Seventh Meeting, June 8th, 1900.

R. F. Muirhead, Esq., M.A., B.Sc., President, in the Chair.

\title{
Note on Proofs by Projection in Trigonometry and Coordinate Geometry.
}

By Prof. George A. Gibson.

The difficulty that is often felt by beginners as to the generality of the proof by projection of the addition theorem in trigonometry and of the formulae for change of axes in coordinate geometry seems to me to be due chiefly to the incomplete treatment of the fundamental theorems on projection. I do not mean that the proofs in the text-books are inaccurate, but only that sufficient pains are not al ways taken to show how the projection of a directed segment is to be measured so that the generality of subsequent proofs shall be beyond all question.

Let $O Q$ be a straight line and $O P Q$ a broken line joining the points $O$ and $Q$. If $\mathrm{P}^{\prime}, Q^{\prime}$ be the projections of $P, Q$ on any line $X^{\prime} O X$ passing through $O$, we have for all positions of $P^{\prime}, Q^{\prime}$, regard being had to the sign $x^{\prime} \quad 0$ of the segments on the axis $\mathrm{X}^{\prime} \mathrm{OX}, \quad \mathrm{OQ}=\mathrm{Q}^{\prime}=\mathrm{P}^{\prime}+\mathrm{P}^{\prime} \mathrm{Q}^{\prime}$.

Let us denote by $(O X, A B)$ the angle which the line $A B$ measured in the direction from $A$ to $B$ makes with the line $O X$ measured from $O$ towards $X$, i.e., the angle through which a line coinciding with OX has to be turned (positively or negatively) till it becomes parallel to and in the same sense as $\mathbf{A B}$; with this convention (OX, BA) differs from (OX, $\mathrm{AB})$ by $180^{\circ}$. If now $\mathrm{OP}, \mathrm{PQ}, \mathrm{OQ}$ are positive segments, then by the definition of the cosine $O Q^{\prime}=O Q \cos (O X, O Q), O P^{\prime}=O P \cos (O X, O P), P^{\prime} Q^{\prime}=P Q \cos (O X, P Q)$, and therefore equation (1) may be written

$$
O Q \cos (O X, O Q)=O P \cos (O X, O P)+P Q \cos (O X, P Q) \text {. }
$$


But if one or more of the lines $O P, P Q, O Q$ be not positive, equation (2) is no longer true as it stands, for in defining the cosine of an angle it is explicitly stated that the radius-vector forming one of the bounding arms of the angle (that is, in the case of the three angles in equation (2) the lines $O Q, O P, P Q$ ) is to be considered positive; manifestly, since equation (2) holds when $\mathrm{OQ}, \mathrm{OP}, \mathrm{PQ}$ are expressed by means of positive numbers, it will no longer be true if one of them be expressed by a negative number-unless some further change be made. It is in the treatment of this phase of the question that I think our text-books should be a little more explicit.

The additional explanation required is, of course, easy to give. When a segment has a sign, then the positive direction of the line on which the segment is taken must have been previously fixed; all that is required to adapt equation (2) to the case of negative segments is to take as the angle which the segment makes with the axis the angle which the positive direction of the line makes with the axis. Thus if $O P$ is a negative segment, we have

$$
\begin{aligned}
\mathrm{OP}^{\prime} & =(-\mathrm{OP}) \times \quad \cos (\mathrm{OX}, \mathrm{OP}), \text { since }(-\mathrm{OP}) \text { is positive } \\
& =(-\mathrm{OP}) \times[-\cos (\mathrm{OX}, \mathrm{PO})], \text { since }(\mathrm{OX}, \mathrm{PO})=(\mathrm{OX}, \mathrm{OP})+180^{\circ} \\
& =\mathrm{OP} \cos (\mathrm{OX}, \mathrm{PO})
\end{aligned}
$$

or, in words, the projection of a directed segment on a directed line is measured by the product of the number which measures the segment and the cosine of the angle which the positive direction of the segment makes with the positive direction of the line.

The general theorem corresponding to equation (2) may then be stated thus:-If $O A B \ldots K L$ be a broken line joining the points $\mathrm{O}, \mathrm{L}$ and $\alpha, \beta, \ldots \lambda, \omega$ the angles which the positive directions of the lines $\mathrm{OA}, \mathrm{AB}, \ldots \mathrm{KL}, \mathrm{OL}$ make with the positive direction of an axis $\mathrm{X}^{\prime} \mathrm{OX}$, and if $a, b, \ldots l, x$ measure the segments $\mathrm{OA}, \mathrm{AB} \ldots \mathrm{KL}, \mathrm{OL}$ in magnitude and in sign, then

$$
x \cos \omega=a \cos \alpha+b \cos \beta+\ldots+l \cos \lambda .
$$

In applying the theorem in trigonometry or in coordinate geometry the chief point requiring attention is that of the positive direction of the segments; when that has been settled there is no difference in carrying out the proof for different figures.

The method of projections is so fundamental in trigonometry and coordinate geometry that no pains should be spared to make its foundations clear. 